\title{
Barakat Syndrome
}

National Cancer Institute

\section{Source}

National Cancer Institute. Barakat Syndrome. NCI Thesaurus. Code C130983.

A condition characterized by hypoparathyroidism, sensorineural deafness, and renal

failure. It is related to autosomal dominant inactivating mutation(s) in GATA3, encoding a transcription factor important for the embryonic development of the parathyroid gland, the auditory stem, and the kidneys. 